\title{
Imaging interferometry to measure surface rotation field
}

\author{
Travaillot, Thomas; Dohn, Søren; Boisen, Anja; Amiot, Fabien
}

Published in:

Applied Optics

Publication date:

2013

Document Version

Publisher's PDF, also known as Version of record

Link back to DTU Orbit

Citation (APA):

Travaillot, T., Dohn, S., Boisen, A., \& Amiot, F. (2013). Imaging interferometry to measure surface rotation field. Applied Optics, 52(18), 4360-4369 .

\section{General rights}

Copyright and moral rights for the publications made accessible in the public portal are retained by the authors and/or other copyright owners and it is a condition of accessing publications that users recognise and abide by the legal requirements associated with these rights.

- Users may download and print one copy of any publication from the public portal for the purpose of private study or research.

- You may not further distribute the material or use it for any profit-making activity or commercial gain

- You may freely distribute the URL identifying the publication in the public portal

If you believe that this document breaches copyright please contact us providing details, and we will remove access to the work immediately and investigate your claim. 


\title{
Imaging interferometry to measure surface rotation field
}

\author{
Thomas Travaillot, ${ }^{1, *}$ Søren Dohn, ${ }^{2}$ Anja Boisen, ${ }^{2}$ and Fabien Amiot ${ }^{1}$ \\ ${ }^{1}$ FEMTO-ST Institute, CNRS-UMR 6174/UFC/ENSMM/UTBM, 24 chemin de l'Épitaphe, Besançon F-25030, France \\ ${ }^{2}$ Nanotech, Technical University of Denmark, Building 345 East, Kongens Lyngby DK-2800, Denmark \\ ${ }^{*}$ Corresponding author: thomas.travaillot @ femto-st.fr
}

Received 18 March 2013; accepted 6 May 2013; posted 21 May 2013 (Doc. ID 187103); published 19 June 2013

\begin{abstract}
This paper describes a polarized-light imaging interferometer to measure the rotation field of reflecting surfaces. This setup is based on a homemade prism featuring a birefringence gradient. The arrangement is presented before focusing on the homemade prism and its manufacturing process. The dependence of the measured optical phase on the rotation of the surface is derived, thus highlighting the key parameters driving the sensitivity. The system's capabilities are illustrated by imaging the rotation field at the surface of a tip-loaded polymer specimen. (C) 2013 Optical Society of America

OCIS codes: (110.3175) Interferometric imaging; (160.2710) Inhomogeneous optical media; (180.3170) Interference microscopy.

http://dx.doi.org/10.1364/AO.52.004360
\end{abstract}

\section{Introduction}

With the miniaturization of devices, microelectromechanical systems development, or more generally with constant advances in thin coating technology, knowledge of the mechanical properties of the involved materials is becoming an important topic in engineering science. The elastic properties of thin films are known to be very dependent on the processing conditions. Their knowledge is thus essential to guarantee the capabilities of systems using these materials, and it is crucial to be able to access the mechanical properties of thin film materials as deposited-that is, without any additional processing. Several methods are available to measure the elastic properties of an isotropic thin film material (described by its Young modulus $E$ and Poisson ratio $\nu$ ). But many of them, such as nanoindentation [1], atomic force acoustic microscopy (AFAM, [2]), and single-mode scanning microdeformation microscopy (SMM, [3-7]), only provide a combination of properties

$1559-128 \mathrm{X} / 13 / 184360-10 \$ 15.00 / 0$

(C) 2013 Optical Society of America
$\left[E /\left(1-\nu^{2}\right)\right.$ for the nanoindentation]. Basically, this latter microscope uses a cantilever, with a microtip (radius $\sim 10 \mu \mathrm{m}$ ) at its end, which vibrates in permanent contact with the sample to characterize. Pressing the tip onto the sample shifts the first resonance frequency according to a combination of $\left[E /\left(1-\nu^{2}\right)\right]^{2 / 3}$ and $\left[E /\left(1-\nu^{2}\right)\right]^{2 / 3} \times(1-\nu) /(2-\nu)$. One thus has to impose the value of $\nu$ to retrieve $\left(E /\left(1-\nu^{2}\right)\right)^{2 / 3}$. Obtaining a full parameter set for isotropic materials and moving toward anisotropic materials is thus challenging.

It is possible to decouple the elasticity constants by combining techniques [믈 10 ] or by using twomodes SMM [11]. In this last case, the parameter driving the Poisson ratio sensitivity is, however, very dependent on a geometrical parameter that is rather difficult to access experimentally from resonance frequencies. Additional experimental information is thus required in order to make the decoupling procedure robust. This could be tip-independent kinematic information such as the out-of-plane displacement field in the neighborhood of the tip. Many imaging interferometric systems are virtually usable to access a displacement field, such as compensated 
interferometers described by Françon or Nomarski employing a Savart polariscope or a Wollaston prism, respectively [12]. It is worth noting that the latter features a usually overlooked tilt sensitivity [13]. It should, however, be noted that the out-of-plane displacement amplitude under the tip is usually a few nanometers or less, so that it may become difficult to access a reliable displacement field. The elastically affected zone being rather small, it would seem wise to measure the rotation field instead of the out-of-plane displacement field. This can be justified considering the problem of Boussinesq [14]: applying a point loading on an elastic half space, the surface displacement field $w(r)$ varies as $1 / r$ (with $r$ : the in-plane distance between the loading point and the point of interest) and is therefore very confined. The surface rotation $\theta(r)$ thus scales as $w(r) / r$ and may reach experimentally accessible values since $r$ is very small. The same applies to vanishingly small structures such as microcantilevers: considering a clamped-free beam (length $L)$ with a constant curvature [15], the maximum out-of-plane displacement scales as $L^{2}$ while the rotation scales as $L$. As a consequence, if $L$ decreases, the out-of-plane displacement decreases faster than the rotation. In the case of a micro- or even a nanosystem, it thus becomes interesting to measure a rotation rather than a displacement.

Many interferometric [16-19] or noninterferometric $[20,21]$ angular measurement systems have been devised in the past to allow for a pointwise measure. Imaging techniques to obtain surface rotation images such as deflectometry [22] or shearography [23] are available at the macroscale.

This paper describes a polarized-light imaging interferometer derived from the one already proposed to measure out-of-plane displacement fields [13]. The originality of the setup is to be based on a homemade prism featuring a birefringence gradient that allows us to measure full rotation (instead of displacement) fields. The device and the interference pattern are described. The manufacturing process for the prism is then detailed. The dependence of the measured optical phase on the rotation of the surface is exhibited, and the key parameters driving its rotation sensitivity are highlighted. An example for practical calibration of the setup is given. Finally, an example with a microtip pressing onto a polymer (PDMS) sample demonstrates the ability of the proposed setup, combined with a phase-stepping method, to catch localized phenomena. Detailed calculations describing the effect of the numerical aperture are presented in Appendix A.

\section{Rotation Field Measurement}

\section{A. Experimental Setup}

A schematic view of the interferential microscopy imaging setup is shown in Fig. 1 . The device is based on a homemade prism, containing a uniaxial birefringence gradient. The light source is a spatially incoherent light-emitting diode (LED, $\lambda=627 \mathrm{~nm}$ ),

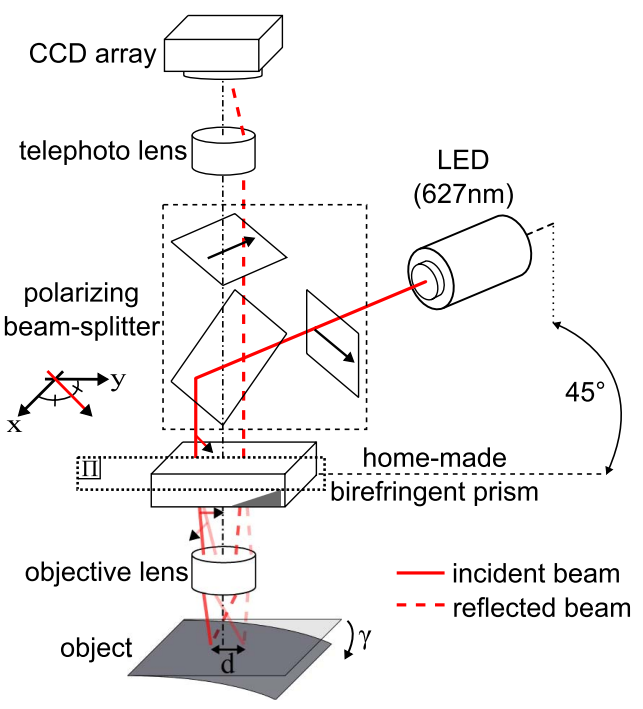

Fig. 1. Schematic view of the interferometric imaging setup.

which is used to illuminate a polarizing beam splitter. After the polarizing beam splitter, the incident beam on the prism is linearly polarized at $45^{\circ}$ of the gradient direction $(\mathbf{y})$ of the birefringent prism. The prism splits the beam into two orthogonally polarized beams with a small angle between them. One of these beams is polarized orthogonally to the plane $\Pi$ defined by the optical axis of the system and the gradient direction of the prism $(\mathbf{y})$, and will be referred to as the transverse electric (TE) beam. The other is polarized in the plane $\Pi$, and will be referred to as the transverse magnetic (TM) beam. These beams are focused upon the sample by an objective lens. After reflection on the sample and recombination by the birefringent prism, the beam goes through the polarization beam splitter, which thus behaves as a polarizer orthogonal to the entrance one. The transmitted beam is finally focused on a CCD array (DALSA 1M30, $1024 \times 1024$ pixels, 12 bits), which records the interference pattern. As the setup is illuminated using an LED, the interference pattern reads [13]

$$
I=I_{0}+A \cos (\phi+\pi)
$$

with $\phi$ the phase shift between the TM and TE polarization components.

\section{B. Homemade Birefringent Prism}

\section{Manufacturing Process}

The key element in the imaging setup is the birefringent prism. The $\left(O_{1} x y\right)$ plane corresponds to the entrance surface of the prism. A heterogeneous stress state is frozen in the material to induce a heterogeneous birefringence state. To set a uniaxial stress gradient of the $\sigma_{x x}$ component along $\mathbf{y}$, it is necessary to induce in the prism a homogeneous bending moment.

In practice, the prism is made out of PS-8A epoxy resin (Vishay Micro-Measurements). A test sample is machined from the polymer plate and then heated up 
above its glass transition temperature $\left(T_{g} \simeq 85^{\circ} \mathrm{C}\right)$. It then undergoes an off-axis tensile test, described in Fig. 2. The test sample is finally cooled at room temperature when maintaining the applied force to freeze the birefringence state in the prism. By tailoring the specimen geometry and loading, it is possible to obtain a wide range of values for the birefringence gradient. Using beam theory, the stress tensor $\underline{\underline{\sigma}}$ in the prism reads

$$
\underline{\underline{\sigma}}=\left(\begin{array}{ccc}
G y & 0 & 0 \\
0 & 0 & 0 \\
0 & 0 & 0
\end{array}\right)_{(x, y, z)},
$$

where $G$ denotes the gradient value.

\section{Refractive Index Field}

For the description of the prism, let us define the $\Pi_{0}$ plane such as $\sigma_{x x}(y)=0$. Let us describe the prism in the plane $\left(O_{1} y z\right)$, where $O_{1}$ is the intersection between the $y$ axis and the plane $\Pi_{0} .\left(O_{1} x z\right)$ and $\left(O_{1} y z\right)$ are assumed to be made coincident with planes $\Pi_{0}$ and $\Pi$, respectively.

The frozen uniaxial stress gradient results in a refractive index gradient in the prism. Initially, the unstressed material has a refractive index $n^{*}=$ 1.5 for both TE and TM rays. As a consequence of the arrangement described in Fig. 2, TM rays are polarized in the plane $\Pi$. As $\sigma_{y y}$ is equal to 0 everywhere, the TM index is equal to $n^{*}$ for any entrance point $\left(n_{\mathrm{TM}}=n^{*}\right)$. TE rays are polarized in the $x$ direction so they experience refractive indices modified by $\sigma_{x x}$. As $\sigma_{x x}$ linearly depends on $y$, the TE index varies linearly with $y$. The TE refractive index thus reads

$$
n_{\mathrm{TE}}=n^{*}+C_{b} G y=n^{*}+c_{\mathrm{TE}} y,
$$

where $C_{b}$ is the photoelastic constant of material. Using a $3 \mathrm{~mm}$ thick polymer plate, a birefringence gradient $c_{\mathrm{TE}} \sim 0.1 \mathrm{~m}^{-1}$ is achieved with the chosen material.

\section{Ray Tracing}

Figure 3 shows (in the $\Pi$ plane) the decomposition of an incident ray by the prism into two emerging rays. Assuming that the surrounding refractive index equals 1, the Snell-Descartes laws on the entrance interface of the prism read

$$
\sin \theta_{e}=n_{\mathrm{TM}} \sin \theta_{a_{\mathrm{TM}}}=n_{\mathrm{TE}}\left(y_{e}\right) \sin \theta_{a_{\mathrm{TE}}},
$$

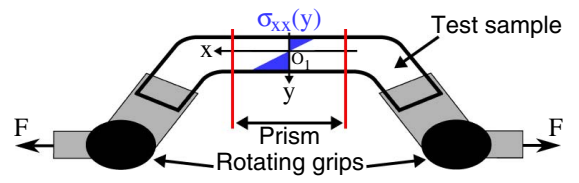

Fig. 2. Arrangement used to establish a stress gradient in the sample. The prism is cut out of the specimen gauge section.

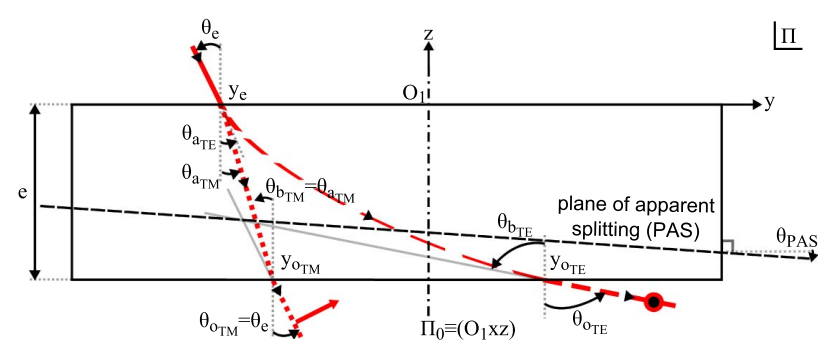

Fig. 3. Ray tracing in the prism.

where $\theta_{e}$ is the incidence angle, $y_{e}$ is the entrance point, and $\theta_{a_{\mathrm{TE}}}$ and $\theta_{a_{\mathrm{TM}}}$ are the angles of the refracted rays at the entrance interface. The Snell-Descartes laws for the exit interface of the prism read

$$
n_{\mathrm{TM}} \sin \theta_{b_{\mathrm{TM}}}=\sin \theta_{o_{\mathrm{TM}}}=\sin \theta_{e}
$$

$$
n_{\mathrm{TE}}\left(y_{o_{\mathrm{TE}}}\right) \sin \theta_{b_{\mathrm{TE}}}=\sin \theta_{o_{\mathrm{TE}}},
$$

where $y_{O_{\mathrm{TE}}}$ is the exit point for the TE ray. $\theta_{b_{\mathrm{TE}}}$ and $\theta_{b_{\mathrm{TM}}}$ are the incidence angles at the exit interface for the TE and TM rays, respectively. $\theta_{O_{\mathrm{TE}}}$ and $\theta_{O_{\mathrm{TM}}}$ are the emerging angles for the TE and TM rays, respectively. The relation between the entrance and exit angles of the prism for the TE ray is given by the eikonal equation and reads

$$
\theta_{b_{\mathrm{TE}}}=\theta_{a_{\mathrm{TE}}}+\epsilon+o\left(\theta_{a_{\mathrm{TE}}}^{3}, \epsilon^{3}\right)
$$

with

$$
\epsilon=\frac{c_{\mathrm{TE}} e}{n_{\mathrm{TE}}\left(y_{e}\right)} \simeq 3 \times 10^{-4} \mathrm{rad}
$$

It depends on the thickness of the prism ( $e=3 \mathrm{~mm}$ ), the birefringence gradient, and the refractive index of the TE ray at the entrance point. $\epsilon$ represents the deflection of the TE ray inside the prism by the birefringence gradient. For the TM ray, the exit point $y_{\mathrm{T}_{\mathrm{TM}}}$ reads

$$
y_{o_{\mathrm{TM}}}=y_{e}+e \tan \theta_{a_{\mathrm{TM}}} .
$$

The relation between entrance and exit points of the prism for the TE ray is also given by the eikonal equation and reads

$$
y_{o_{\mathrm{TE}}}=y_{e}+e\left(\theta_{a_{\mathrm{TE}}}+\frac{\epsilon}{2}\right)+o\left(\theta_{a_{\mathrm{TE}}}^{3}, \epsilon^{3}\right) .
$$

The separation between $\mathrm{TE}$ and $\mathrm{TM}$ rays reads

$$
\theta_{o_{\mathrm{TE}}}-\theta_{o_{\mathrm{TM}}}=c_{\mathrm{TE}} e+o\left(\theta_{e}^{3}, \epsilon^{3}\right) \sim 10^{-4} \mathrm{rad} .
$$

It only depends on the thickness of the prism and the birefringence gradient. Further developments will be made easier by defining the plane of apparent splitting (PAS; see dashed line in Fig. $\underline{3}$ ). For an 
incident ray, it gathers the points where TE and TM rays appear to split. The equation of the PAS reads

$$
z=\tan \left(\theta_{\mathrm{PAS}}\right) y-e\left[1-\frac{1}{2 n^{*}}\right]+o\left(\theta_{e}^{2}, \epsilon^{2},\left(c_{\mathrm{TE}} y_{e}\right)^{2}\right)
$$

with

$$
\theta_{\mathrm{PAS}}=\frac{e c_{\mathrm{TE}}+2 \theta_{e}}{2 n^{*^{2}}}+o\left(\theta_{e}^{2}, \epsilon^{2},\left(c_{\mathrm{TE}} y_{e}\right)^{2}\right)
$$

$\theta_{\text {PAS }}$ is the angle between the PAS and the surface of the prism (see Fig. 3). Taylor expansion with respect to $c_{\mathrm{TE}} y_{e}$ is possible because $c_{\mathrm{TE}} y_{e} \ll n^{*}$ in the expression of $\epsilon$ [see Eq. ( $\underline{8})]$.

\section{Optical Path Length in the Prism}

For the TM ray, the refractive index is constant, so the trajectory is rectilinear and the optical length $L_{\mathrm{TM}}$ reads

$$
L_{\mathrm{TM}}=n^{*} \frac{e}{\cos \theta_{a_{\mathrm{TM}}}}=\frac{n^{*^{2}} e}{\sqrt{n^{*^{2}}-\sin ^{2} \theta_{e}}} .
$$

The optical path length for the TM ray thus depends on the incidence angle (see Fig. 3) but does not depend on the entrance point. For the TE ray, the optical path length reads

$$
L_{\mathrm{TE}}=\int_{-e}^{0} \frac{n_{\mathrm{TE}}\left(y_{\mathrm{TE}}(z)\right)}{\cos \left(\theta_{\mathrm{TE}}(z)\right)} \mathrm{d} z
$$

with

$$
\begin{gathered}
y_{\mathrm{TE}}(z)=y_{e}-z\left(\frac{\theta_{e}}{n_{\mathrm{TE}}\left(y_{e}\right)}-\frac{\epsilon z}{2 e}\right)+o\left(\theta_{e}^{3}, \epsilon^{3}\right), \\
\theta_{\mathrm{TE}}(z)=\frac{\theta_{e}}{n_{\mathrm{TE}}\left(y_{e}\right)}-\frac{\epsilon z}{e}+o\left(\theta_{e}^{3}, \epsilon^{3}\right) .
\end{gathered}
$$

The optical path length for the TE ray finally reads

$$
\begin{aligned}
L_{\mathrm{TE}}= & e\left[n_{\mathrm{TE}}\left(y_{e}\right)\left(1+\frac{\epsilon^{2}}{6}\right)+\frac{c_{\mathrm{TE}} e}{6} \epsilon+\epsilon \theta_{e}+\frac{\theta_{e}^{2}}{2 n_{\mathrm{TE}}\left(y_{e}\right)}\right] \\
& +o\left(\theta_{e}^{3}, \epsilon^{3}\right) .
\end{aligned}
$$

$L_{\mathrm{TE}}$ depends on both the incidence angle $\theta_{e}$ and the entrance point $y_{e}$.

\section{Optical Phase}

Let us assume that the optical phase difference $\phi$ due to the path prism-objective-sample-objective prism can be decomposed as $\phi=\phi_{p}+\phi_{o}$, where $\phi_{p}$ denotes the part arising from the birefringent prism and $\phi_{o}$ denotes the contribution arising from the object.

\section{Optical Phase Arising from the Birefringent}

Prism

Figure 4 presents the full ray tracing for the two emerging rays of Fig. 3 . $\delta_{\mathrm{PAS}}$ and $\alpha_{\mathrm{PAS}}$ are the position and the rotation, respectively, of the actual PAS with respect to the rear focal plane of the objective. The point $\mathrm{O}_{2}$ is the intersection of the PAS with the optical axis. It is the origin of the frame $\left(O_{2} Y Z\right): Z$ is made coincident with the optical axis and $Y$ lies in the $\Pi$ plane. $\Delta$ is the distance between $O_{2}$ and $O_{1}$ projected onto $Y$ (if $\Delta=0, O_{1}$ and $O_{2}$ are on the optical axis). $\gamma_{\mathrm{TE}}$ and $\gamma_{\mathrm{TM}}$ define the surface orientation for $\mathrm{TE}$ and TM rays, respectively. The two rays emerge from the PAS at the point whose orthogonal projection on the $Y$ axis is $Y_{\text {PAS. Then, they travel through }}$ the objective, are reflected by the sample, and intersect the PAS at $Y_{\mathrm{PAS}}^{\prime}$ and $Y_{\mathrm{PAS}}^{\prime}$ :

$$
\begin{aligned}
Y_{\mathrm{PAS}_{i}}^{\prime}= & -Y_{\mathrm{PAS}}-2 \frac{f_{o}^{2}+Y_{\mathrm{PAS}}^{2}}{f_{o}} \gamma_{i}-2\left[\alpha_{\mathrm{PAS}}+\left(1+\frac{1}{n^{*^{2}}}\right) \theta_{e}\right. \\
& \left.+n^{*} \epsilon+\frac{e c_{\mathrm{TE}}}{2 n^{*^{2}}}\right] \delta_{\mathrm{PAS}}+o\left(\alpha_{\mathrm{PAS}}^{2}, \theta_{e}^{2}, \theta_{\mathrm{PAS}}^{2}, \epsilon^{2}, \gamma_{i}^{2}\right),(19)
\end{aligned}
$$

where $i$ stands for TE or TM.

The position of $Y_{\mathrm{PAS}_{i}}^{\prime}$ depends on the position of $Y_{\mathrm{PAS}}$, on the objective focal length $f_{o}$, on the incidence angle $\theta_{e}$, on PAS position ( $\left.\delta_{\mathrm{PAS}}, \alpha_{\mathrm{PAS}}\right)$, and on the corresponding surface orientation $\left(\gamma_{i}\right)$. As described in Eq. (18), the optical path length in the prism for the TE ray depends on the entrance point. For the back TE path, this entrance point $\left(Y_{\mathrm{PAS}_{\mathrm{TE}}^{\prime}}^{\prime}\right)$ depends on $\gamma_{\mathrm{TE}}$, so that the back optical path length in the prism for the TE ray depends on $\gamma_{\mathrm{TE}}$. Equation (14) indicates that the optical path length in the prism for the TM ray only depends on the incidence angle and does not depend on the entrance point. As a consequence, the optical path length in the prism for the TM ray is independent of the TM orientation of the sample $\gamma_{\mathrm{TM}}$. Finally, the total optical phase

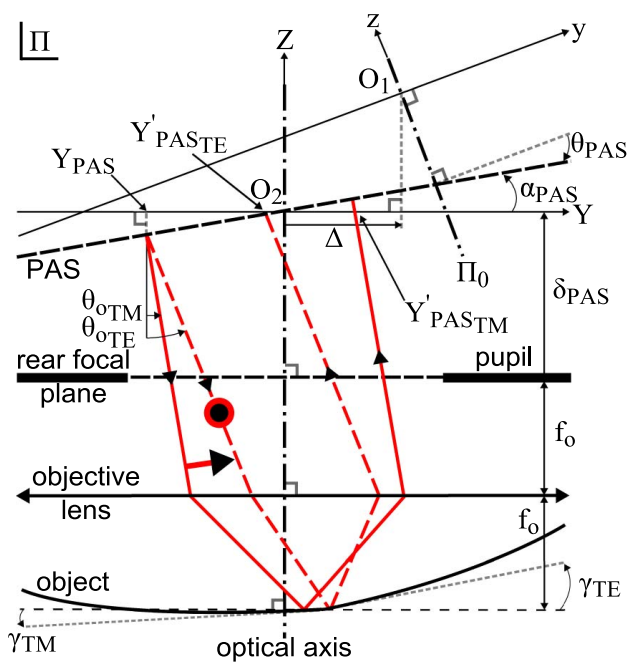

Fig. 4. Ray tracing through the objective for the two emerging rays of Fig. 3 . 
thus depends on $\gamma_{\mathrm{TE}}$. It demonstrates how the system is sensitive to the TE orientation of the sample and insensitive to the TM one. The TM beam will thus act as a reference beam in the interferometer. The total optical path length arising from the birefringent prism is the difference between the optical path lengths for the TE and TM rays:

$$
\begin{aligned}
\phi_{p}\left(\gamma_{\mathrm{TE}}\right)= & \frac{2 \pi}{\lambda}\left\{\left[L_{\mathrm{TE}_{\text {forth }}}+L_{\mathrm{TE}_{\text {back }}}\left(\gamma_{\mathrm{TE}}\right)\right]\right. \\
& \left.-\left[L_{\mathrm{TM}_{\text {forth }}}+L_{\mathrm{TM}_{\text {back }}}\right]\right\} .
\end{aligned}
$$

Using Eqs. (14), (18), and (20) and first-order Taylor expansion with respect to $\alpha_{\mathrm{PAS}}, \theta_{e}, \theta_{\mathrm{PAS}}, \epsilon$, and $\gamma_{\mathrm{TE}}$, it reads

$$
\phi_{p}=\phi_{\gamma_{\mathrm{TE}}}+\phi_{\theta_{e}}+\phi_{\Delta}+\phi_{r}
$$

with

$$
\begin{gathered}
\phi_{\gamma_{\mathrm{TE}}}=-\frac{4 \pi}{\lambda} c_{\mathrm{TE}} e \frac{f_{o}^{2}+Y_{e}^{2}}{f_{o}} \gamma_{\mathrm{TE}}+o\left(\gamma_{\mathrm{TE}}^{2}\right), \\
\phi_{\theta_{e}}=-\frac{2 \pi}{\lambda} c_{\mathrm{TE}} e\left[2\left(1+\frac{1}{n^{*^{2}}}\right) \delta_{\mathrm{PAS}}+\frac{e}{n^{*}}\right] \theta_{e}+o\left(\theta_{e}^{2}, \theta_{\mathrm{PAS}}^{2}\right), \\
\phi_{\Delta}=-\frac{4 \pi}{\lambda} c_{\mathrm{TE}} e \Delta+o\left(\theta_{\mathrm{PAS}}^{2}\right) \\
\left.+\left(2 n^{*} \delta_{\mathrm{PAS}}-\frac{e}{3}\right) \epsilon\right]+o\left(\alpha_{\mathrm{PAS}}^{2}, \theta_{\mathrm{PAS}}^{2}, \epsilon^{2}\right),
\end{gathered}
$$

where $Y_{e}$ denotes the entrance point on the prism (on the $Y$ axis). $\Delta$ can be changed by translating the prism so it will be used for phase modulation.

The rotation sensitivity $\partial \phi / \partial \gamma_{\mathrm{TE}}$ depends on the thickness of the prism $e$ and on the birefringence gradient $c_{\mathrm{TE}}$. It also increases with the objective focal length $f_{o}$ and with the prism entrance point $Y_{e}$. So, Eq. (21) is only valid for a ray. For the full beam, the rotation sensitivity thus depends on the objective numerical aperture (see Appendix A). In addition, expanding Eq. (21) up to the second-order shows the rotation sensitivity dependence on the incidence angle and on the implementation defects is negligible.

Finally, the lateral shear $d$ between TE and TM rays on the sample (see Fig. 1 ) reads

$$
d=f_{o} c_{\mathrm{TE}} e+o\left(\theta_{e}^{2}, \theta_{\mathrm{PAS}}^{2}, \alpha_{\mathrm{PAS}}^{2}, \epsilon^{2}\right) .
$$

It depends on the objective focal length $f_{o}$ as well as on the thickness of the prism and birefringence gradient. $d$ corresponds to the separation, in the $Y$ direction, between the two reflected images of the sample due to the birefringence.

\section{Optical Phase Arising from the Object}

In addition to $\phi_{p}$, there is also a phase contribution arising from the object. Let us consider the case of a tilted and stepped sample (height $\Delta Z=Z_{\mathrm{TE}}-Z_{\mathrm{TM}}$ ), where TE and TM rays are reflected at different heights. According to the principle of Fermat, tilting the sample does not induce any additional phase difference in the objective-sample-objective path. However, the step induces an additional phase $\phi_{0}$, which reads (assuming the ambient refractive index of the medium is 1 )

$$
\phi_{o}=-\frac{4 \pi}{\lambda} \Delta Z \cos \alpha,
$$

where $\alpha$ is the incidence angle on the sample. $\alpha$ spans the full range defined by the objective pupil, so that for the full beam $\phi_{o}$, an integration over $\alpha$ has to be considered (see Appendix $\underline{\mathrm{A}}$ ).

\section{Calibration and Example}

As the parameters driving the phase sensitivity to the topography depend on the numerical aperture and thus on the illumination, a calibration procedure is desirable for practical applications. This section presents the calibration experiment of the setup and an example to validate both the system capabilities and its modeling.

\section{A. Calibration Experiment}

The calibration consists in plotting interferograms obtained by tilting a plane sample. Namely, a PDMS sample, charged with 50 wt. \% of Co nanoparticles, is tilted from $\delta \gamma=-5^{\circ}$ to $5^{\circ}$ by $0.05^{\circ}$ steps with respect to the (unknown) initial stage orientation $\gamma_{d}$. Intensity images are acquired for each tilt value. This experiment gives one interferogram per pixel, which are used to retrieve modeling parameters. For the calibration, the phase equation for one ray $(\underline{\mathrm{A} 2})$ is recast:

$$
\phi\left(K, \gamma_{d}+\delta \gamma, \Psi, \alpha\right)=-K\left[1+\sin ^{2}(\alpha)\right]\left[\gamma_{d}+\delta \gamma\right]+\Psi
$$

with

$$
\begin{gathered}
K=\frac{4 \pi}{\lambda} c_{\mathrm{TE}} e f_{o}, \\
\gamma_{d}+\delta \gamma=\gamma_{\mathrm{TE}}=\gamma_{\mathrm{TM}}, \\
\Psi=\phi_{\theta_{e}}+\phi_{\Delta}+\phi_{r} .
\end{gathered}
$$

$\Psi$ represents the phase contribution independent of $\gamma_{\mathrm{TE}}$, as the contribution (27) from the object vanishes in this configuration. Because of the $\theta_{e}$ dependence, $\Psi$ reads 


$$
\Psi=\Psi_{a} Y+\Psi_{b}
$$

with

$$
\Psi_{a} Y=\phi_{\theta_{e}}, \quad \Psi_{b}=\phi_{\Delta}+\phi_{r}
$$

Let us denote NA the numerical aperture of the objective. Taking the full aperture into account, Eq. (1) is modified by weighting and summing all useful rays of the light beam (see Appendix A), and the intensity equation $(\underline{\mathrm{A} 4})$ reads

$$
\begin{aligned}
& I\left(K, \gamma_{d}+\delta \gamma, \Psi, \gamma_{c}, \mathrm{NA}, m\right)=I_{0} \\
& \quad+A F\left(\gamma_{d}+\delta \gamma, \gamma_{c}, \mathrm{NA}, m, K, \Psi\right)
\end{aligned}
$$

$F$ is the weighted sum of the contribution of all useful rays (some light rays are lost in the pupil of the objective, depending on the tilt). $m$ is a parameter used to describe the pupil illumination. It is used in the apodization function $P_{m}$, which corresponds to the repartition of light on the pupil $\left(P_{m}(\alpha)=[\cos (\alpha)]^{m}\right) \cdot \gamma_{c}$ is used to account for negligible phenomena not taken into consideration in the model, such as the distance between the pupil and the rear focal plane. It modifies the pseudoperiod of the intensity with the tilt. For the sake of generality, $\gamma_{c}$ is assumed to depend on $Y$ :

$$
\gamma_{c}=\gamma_{c_{a}} Y+\gamma_{c_{b}} .
$$

The involved parameters thus fall into two categories. The first one contains global parameters (parameters that have the same value for all pixels): $K, \gamma_{d}$, NA, $m, \Psi_{a}, \Psi_{b}, \gamma_{c_{a}}$, and $\gamma_{c_{b}}$. The second one contains the local parameters (parameters that have a different value for each pixel): $I_{0}$ and $A$. Starting with a set of global parameters $p$, the first step of the identification procedure consists in calculating $F(p, \delta \gamma)$ for all values of $\delta \gamma$. For each pixel $(i, j)$, one thus defines the local residual:

$$
\begin{aligned}
R_{0}^{2}\left(p, I_{0}(i, j), A(i, j)\right)= & \sum_{\delta \gamma}\left\{I_{\exp }(i, j, \delta \gamma)\right. \\
& \left.-\left[I_{0}(i, j)+A(i, j) F(p, \delta \gamma)\right]\right\}^{2}
\end{aligned}
$$

The optimal values $I_{0_{\text {optimum }}}(i, j)$ and $A_{\text {optimum }}(i, j)$ are obtained as the minimizers of the residual $R_{0}^{2}$. Using more than two different $\delta \gamma$ values, the stationarity condition yields an overdetermined linear system for each pixel. The description quality is then locally assessed through

$$
R_{1}^{2}(p, i, j)=\min _{I_{0}(i, j), A(i, j)} R_{0}^{2}\left(p, I_{0}(i, j), A(i, j)\right)
$$

A global residual taking into consideration residuals $R_{1}^{2}$ for every pixel is then defined:

$$
R_{2}^{2}(p)=\frac{\sum_{i, j} R_{1}^{2}(p, i, j)}{\sum_{i, j} \sum_{\delta \gamma}\left[I_{\exp }(i, j, \delta \gamma)\right]^{2}} .
$$

The set of parameters $p_{\text {optimum }}$ is retrieved as the minimizer of $R_{2}^{2}$, using the conjugate gradient algorithm. Let us define the final residual, which reads

$$
R_{3}^{2}=\min _{p} R_{2}^{2}(p)
$$

$p_{\text {optimum }}, I_{0_{\text {optimum }}}(i, j), A_{\text {optimum }}(i, j)$ is then the set of fitted parameters. This calibration has to be performed after each modification of the setup.

The interferograms for three different pixels, along the $Y$ direction, are displayed in Fig. 5 . The position of the interferogram center is driven by $\Psi$. The attenuation of the signal with absolute tilt (upper envelope) is driven by NA, $m$, and $\gamma_{d}$. The pseudoperiod is driven by NA, $K$, and $\gamma_{c}$. The nominal and the experimentally identified global and local parameter values, all calculated on 160 pixels distributed regularly along the two lines of Fig. 6 ( 80 regularly spaced pixels by line), are given in Tables 1 , $\underline{2}$, and $\underline{3}$, respectively. The comparison between the fitted and the experimental values shows that the fitted value of NA is the same as the experimental one within $2.4 \%$. $m$ is almost 0 , so the repartition of light on the pupil is almost homogeneous. The retrieved

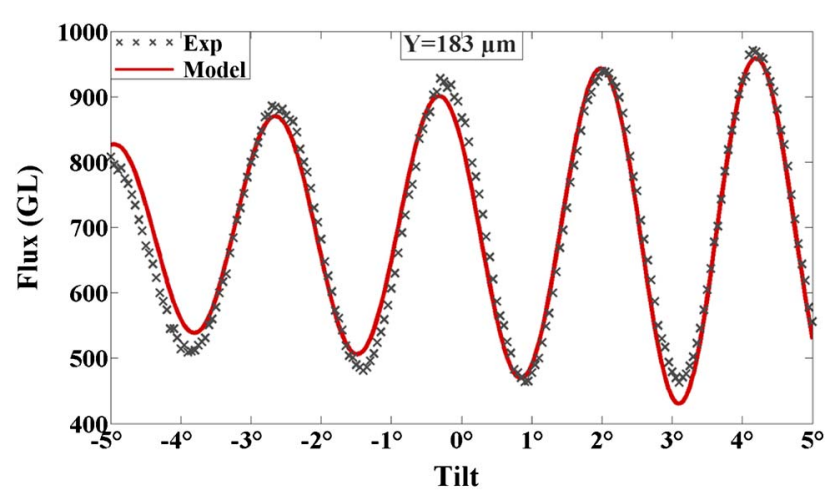

(a)

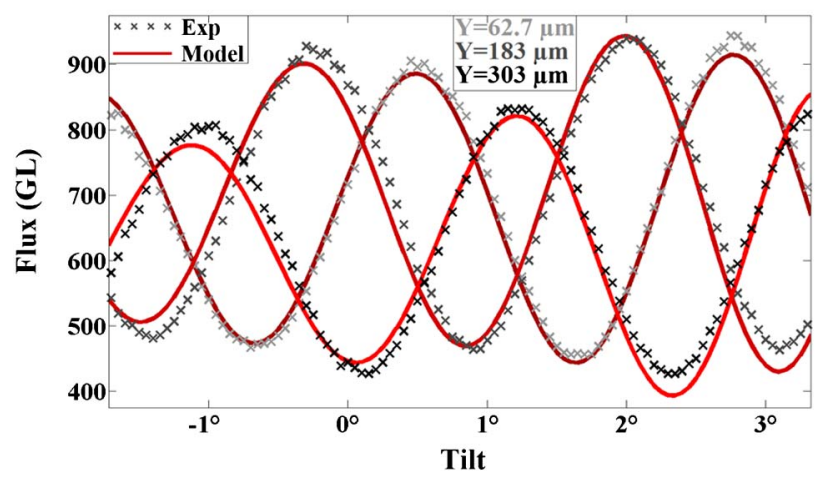

(b)

Fig. 5. Tilting sample interferograms. (a) For $Y=183 \mu \mathrm{m}$ and for $\delta \gamma$ ranging from $-5^{\circ}$ to $5^{\circ}$. (b) For $Y=\{62.7,183,303\} \mu \mathrm{m}$ and for $\delta \gamma$ ranging from $-2^{\circ}$ to $3^{\circ}$. 


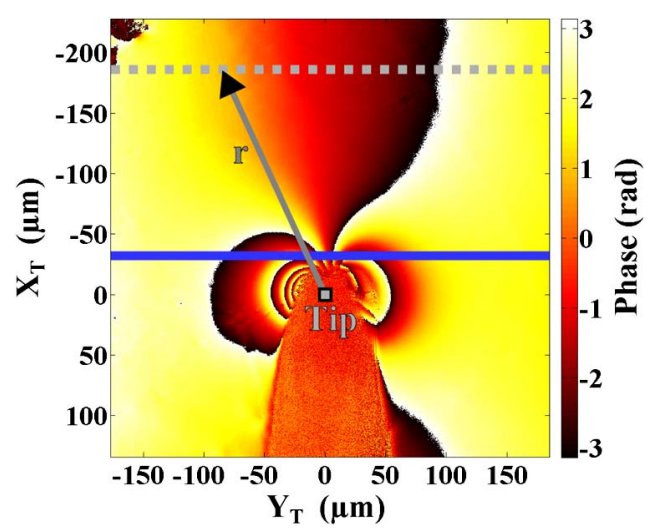

Fig. 6. Phase map obtained when pressing a tip onto a PDMS sample.

value for $K$ is lower than the estimated one; it is, to a large extent, due to $c_{\mathrm{TE}}$, which is estimated with an uncertainty of almost $10 \%$. The identified initial stage misorientation $\gamma_{d}$ is about $3.7^{\circ}$, which is a realistic value because the surfaces of the sample are not parallel. Finally, the residual $R_{3}^{2}$ is about $2.31 \times 10^{-3}$, thereby proving the identification quality.

\section{B. Phase Modulation Calibration}

The $\Delta$ sensitivity $\left(s_{\Delta}=\partial \phi / \partial \Delta\right)$ will be used in Subsection 3.C for phase modulation and has thus to be estimated. It is proposed to change $\Delta$ from a known value $\delta \Delta$ (here, $\delta \Delta=0.5 \mathrm{~mm}$ ) and to reproduce the above-described calibration experiment. For this second calibration, global parameters are set to the previously identified values $p_{\text {optimum }}$ (obtained in the previous section), except the parameter $\Psi_{b}$, which is changed to $\Psi_{b}^{\prime}$ :

$$
\Psi_{b}^{\prime}=\phi_{\Delta+\delta \Delta}+\phi_{r}=\Psi_{b}+\phi_{\delta \Delta}=\Psi_{b}+s_{\Delta} \delta \Delta .
$$

$\Psi_{b}$ is the homogeneous part of the $\gamma_{\mathrm{TE}}$-independent contribution of the phase. The calibration procedure is the same as in the previous section, but the last

Table 1. Estimated Parameters

\begin{tabular}{ccc}
\hline LED & \multicolumn{2}{c}{ Objective } \\
\hline$\lambda(\mathrm{nm})$ & $f_{o}(\mathrm{~mm})$ & $\mathrm{NA}$ \\
627 & 20 & 0.45 \\
\hline \multicolumn{2}{c}{ Prism } & Rotation sensitivity \\
\hline$c_{\mathrm{TE}}\left(\mathrm{m}^{-1}\right)$ & $e(\mathrm{~mm})$ & $K\left(\mathrm{rad} \cdot \mathrm{rad}^{-1}\right)$ \\
0.139 & 3 & \multicolumn{2}{c}{167} \\
\hline
\end{tabular}

Table 2. Fitted Global Parameters

\begin{tabular}{lcc}
\hline NA & $m$ & $K\left(\mathrm{rad} \cdot \mathrm{rad}^{-1}\right)$ \\
0.439 & $2.78 \times 10^{-4}$ & 147 \\
\hline$\gamma_{c_{a}}\left(\mathrm{rad} \cdot \mu \mathrm{m}^{-1}\right)$ & $\gamma_{c_{b}}(\mathrm{rad})$ & $\gamma_{d}(\mathrm{rad})$ \\
$2.30 \times 10^{-4}$ & 0.117 & $-6.45 \times 10^{-2}$ \\
\hline$\Psi_{a}\left(\mathrm{rad} \cdot \mu \mathrm{m}^{-1}\right)$ & $\Psi_{b}(\mathrm{rad})$ & $\mathrm{R}_{3}^{2}$ \\
$-1.87 \times 10^{-2}$ & 4.82 & $2.31 \times 10^{-3}$ \\
\hline
\end{tabular}

Table 3. Fitted Local Parameters

\begin{tabular}{lcccccc}
\hline Interferogram $X(\mu \mathrm{m})$ & $Y(\mu \mathrm{m})$ & $I_{0}(\mathrm{GL})$ & $A(\mathrm{GL})$ & $\Psi(\mathrm{rad})$ & $\gamma_{c}(\mathrm{rad})$ \\
\hline (a) & 42.5 & 62.7 & 672 & 234 & 3.65 & 0.132 \\
(b) & 42.5 & 183 & 695 & 251 & 1.40 & 0.160 \\
(c) & 42.5 & 303 & 620 & 224 & -0.853 & 0.187 \\
\hline
\end{tabular}

minimization is performed with respect to $\Psi_{b}^{\prime}$ instead of the full set $p$. For the actual setup (experimental parameters values are given in Table $\underline{1}$ ), one obtains

$$
\begin{gathered}
s_{\Delta_{\mathrm{th}}}=-\frac{4 \pi}{\lambda} c_{\mathrm{TE}} e \simeq-8.36 \times 10^{3} \mathrm{rad} \cdot \mathrm{m}^{-1}, \\
s_{\Delta_{\exp }}=\frac{\Psi_{b}^{\prime}-\Psi_{b}}{\delta \Delta} \simeq-6.66 \times 10^{3} \mathrm{rad} \cdot \mathrm{m}^{-1}
\end{gathered}
$$

The difference between the theoretical and the experimental values is, to a large extent, due to $c_{\mathrm{TE}}$ and is consistent with the error on $K$ :

$$
\begin{gathered}
K_{\mathrm{th}}=\frac{4 \pi}{\lambda} c_{\mathrm{TE}} e f_{o} \simeq 167 \mathrm{rad} \cdot \mathrm{rad}^{-1}, \\
K_{\mathrm{exp}} \simeq 147 \mathrm{rad} \cdot \mathrm{rad}^{-1} \\
\frac{K_{\mathrm{th}}}{\left|s_{\Delta_{\mathrm{th}}}\right|}=f_{o}=20 \mathrm{~mm}, \\
\frac{K_{\exp }}{\left|s_{\Delta_{\exp }}\right|} \simeq 22.1 \mathrm{~mm} .
\end{gathered}
$$

The value of $\Delta$ sensitivity can be compared with the parameter $K$. The identification is consistent because the ratios (theoretical and experimental) between the two parameters give the same value within $10 \%$, thereby proving the slight discrepancy obtained on $K$ results from the product $c_{\mathrm{TE}} e$. In addition, the value of the shear $d$ [Eq. (26)] is compared with the parameter $K . d$ is obtained by measuring the separation between the two superimposed pictures on an intensity image. One obtains $d_{\exp } \simeq 7.86 \mu \mathrm{m}$, which should be compared to $d_{\mathrm{th}}=c_{\mathrm{TE}} e f_{o}=8.34 \mu \mathrm{m}$. Consider the ratios

$$
\frac{K_{\mathrm{th}}}{d_{\mathrm{th}}}=\frac{4 \pi}{\lambda} \simeq 20.0 \mu \mathrm{m}^{-1},
$$

$$
\frac{K_{\exp }}{d_{\exp }} \simeq 18.7 \mu \mathrm{m}^{-1}
$$

The identification is consistent with a discrepancy in $c_{\mathrm{TE}} e$ because the ratios (theoretical and experimental) between the two parameters give the same value at almost $6.5 \%$. 


\section{Example of Phase Map}

The goal is to measure the rotation field around a tip (radius: $10 \mu \mathrm{m}$ ) that presses onto a sample. The validation experiment consists in pressing a tip onto the specimen described in Subsection 3.A.

The considered phase-stepping method makes use of four pictures obtained for $\Delta_{k}=[0,0.1,0.2,0.3] \mathrm{mm}$. Using Eq. (1) to approximate the intensity (a small range of tilt is swept so interferograms remain similar to a sine curve), the measured intensities read

$$
I_{\text {exp }}\left(i, j, \Delta_{k}\right)=I_{0}(i, j)+A(i, j) \cos \left[\phi(i, j)+s_{\Delta} \Delta_{k}\right] .
$$

Equation (50) therefore yields four equations per pixel for only three unknowns $\left(I_{0}(i, j), A(i, j)\right.$, and $\phi(i, j))$, so that $\phi$ is obtained by solving it in a least-square sense.

A phase map example is presented on Fig. 6 . It is obtained when pressing a tungsten tip (radius $=30 \mu \mathrm{m}$ ) onto an opaque sample of PDMS, charged with 50 wt. \% of Co nanoparticles (sample thickness $\approx 2.6 \mathrm{~mm}$ ). The noise is obtained by making two identical phase maps and averaging the difference between them. The noise on phase $\phi_{\text {noise }}$ is estimated to $8.9 \times 10^{-3} \mathrm{rad}$. The phase map is described in the $\left(O_{T} X_{T} Y_{T}\right)$ plane, with $O_{T}$ the tip loading point. $X_{T}$ and $Y_{T}$ are oriented as $X$ and $Y$. Let us comment on the phase along the $Y_{T}$ axis in two different parts of the phase map. The first one corresponds to the zone far from the tip (dotted line in Fig. 6) where the sample is not deformed. The phase is linear with respect to $Y_{T}$ (circles in Fig. 7), as a result of the dependence on the incidence angle $\left(\theta_{e}\right)$. It corresponds to the parameter $\Psi$ presented in the calibration subsection. The phase equation reads

$$
\phi_{\text {far from the tip }}=\phi_{\theta_{e}}+\phi_{\Delta}+\phi_{r}=\Psi_{a} Y+\Psi_{b}=\Psi .
$$

The theoretical phase far from the tip in Fig. 7 is a plotting of $\Psi$ coming from the calibration subsection, along the $Y_{T}$ axis. The good agreement validates the proposed modeling. The crosses in Fig. $\underline{7}$ correspond

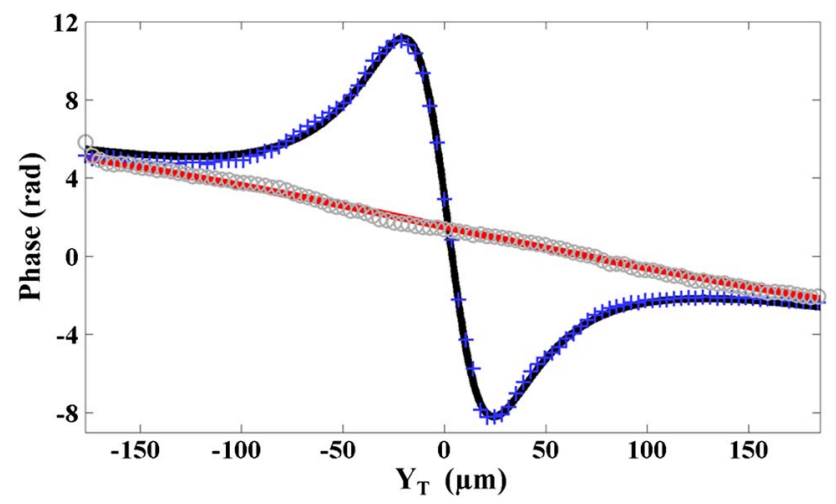

Fig. 7. Phase along $Y_{T}$ axis. Circles, experimental phase far from the tip; crosses, experimental phase close to the tip; solid lines, theoretical phases far and close to the tip. to a zone (solid line in Fig. 6) where the sample is deformed by the tip so the $\gamma_{\mathrm{TE}}$ contribution of the optical phase is activated. The presence of a phase deviation from the previous line in the vicinity of the tip shows the presence of the rotation field in the $Y$ direction. This phase map proves that the system allows one to measure the rotation field of localized phenomena (here, a few tens of micrometers). Let us assume that the situation corresponds to the problem of Boussinesq [14]: an elastic half space with a point load. In this case, the displacement $w$ scales as $w\left(X_{T}, Y_{T}\right)=-P /\left(\pi E^{*} r\right)$ [with $r=\sqrt{X_{T}^{2}+Y_{T}^{2}}$ the in-plane distance between the loading point and the point of interest (see Fig. $\underline{6}$ ), $P$ the loading onto the sample, and $E^{*}=E /\left(1-\nu^{2}\right)$ the biaxial Young's modulus of the sample) and the rotation $\theta$ as $\theta\left(X_{T}, Y_{T}\right)=P /\left(\pi E^{*} r^{2}\right)$. So, the measured rotation field scales as $\gamma_{\mathrm{TE}}\left(X_{T}, Y_{T}\right)=P Y_{T} /\left(\pi E^{*} r^{3}\right)$, and the contribution from the object scales as $\Delta Z\left(X_{T}, Y_{T}, d\right)=w\left(X_{T}, Y_{T}\right)-w\left(X_{T}, Y_{T}-d\right)$, because the measure is only made in the $Y$ direction. Finally, the phase equation reads

$$
\begin{aligned}
\phi_{\mathrm{tip}}= & \phi_{\theta_{e}}+\phi_{\Delta}+\phi_{r}+\phi_{\gamma_{\mathrm{TE}}}+\phi_{o} \\
= & \Psi_{a} Y+\Psi_{b}+\frac{\partial \phi}{\partial \gamma_{\mathrm{TE}}} \gamma_{\mathrm{TE}}\left(X_{T}, Y_{T}\right) \\
& +\frac{\partial \phi}{\partial \Delta Z} \Delta Z\left(X_{T}, Y_{T}, d\right)
\end{aligned}
$$

with $X_{T}$ fixed on the line and $Y_{T}$ variable along the line.

The solution of Boussinesq (solid line close to the tip on Fig. 7) fits the measured phase, thereby demonstrating the ability of the setup to catch a localized phenomenon.

\section{Conclusions}

The proposed setup makes use of a birefringent prism whose fabrication procedure is presented. It allows one to access the rotation field of reflecting surfaces, projected onto a particular prism direction. It must be highlighted that the setup is therefore well suited to localized phenomena. This method is particularly useful for situations in which scale effects make the rotation measurement preferable to the out-of-plane displacement measurement. The noise on the phase measurement is estimated to $8.9 \times 10^{-3}$ rad without any image accumulation. As a consequence, it is thought to be useful to study the deformation of samples in SMM. A detailed modeling including aperture effects is proposed, and a calibration procedure allows one to retrieve the parameters required by a quantitative use of the obtained phase maps. The ability of the setup to catch localized mechanical phenomena is therefore demonstrated. Future work will thus focus on coupling this imaging arrangement with an SMM setup. 


\section{Appendix A: Effect of the Numerical Aperture on the Interferogram}

In Subsections 2.C.1 and 2.C.2, expressions of the optical phase arising from the birefringent prism and from the object were calculated [Eqs. (21) and (27)]. It is shown that, for one ray, the phase depends on the entrance point of the prism $Y_{e}$ and on the incidence angle $\alpha$. This means that, for the full beam, the phase depends on the objective numerical aperture $\left[\mathrm{NA}=\sin \left(\alpha_{\max }\right)\right]$. The sine condition [24] yields

$$
\sin (\alpha)=-\frac{Y_{e}}{f_{o}}+o\left(\theta_{e}^{2}, \alpha_{\mathrm{PAS}}^{2}, \theta_{\mathrm{PAS}}^{2}\right)
$$

so that using Eqs. (21) and (27), the total phase difference reads

$$
\begin{aligned}
\phi\left(\gamma_{\mathrm{TE}}, \alpha\right)= & -\frac{4 \pi}{\lambda} c_{\mathrm{TE}} e f_{o}\left[1+\sin ^{2}(\alpha)\right] \gamma_{\mathrm{TE}} \\
& -\frac{4 \pi}{\lambda} \Delta Z \cos \alpha+\phi_{\theta_{e}}+\phi_{\Delta}+\phi_{r}+o\left(\gamma_{\mathrm{TE}}^{2}\right) .
\end{aligned}
$$

To assess the effect of the numerical aperture, it is necessary to consider the contribution of every angle of incidence [see Fig. 8(a)], based on Eqs. (1) and (A3). The light intensity is obtained by summing the contribution of all useful rays:

$$
I\left(\gamma_{\mathrm{TE}}, \gamma_{c}, \mathrm{NA}, m\right)=I_{0}+A F\left(\gamma_{\mathrm{TE}}, \gamma_{c}, \mathrm{NA}, m\right) .
$$

$F$ is obtained by weighting and summing the contribution of each ray over the numerical aperture. Because of the tilting, a part of the light beam is not collected at the level of the pupil of the objective so that only the useful rays are considered. Useful rays correspond to the surface that is not darkened on Fig. 8(b) and thus define the integration bounds in the following $F$ equations.

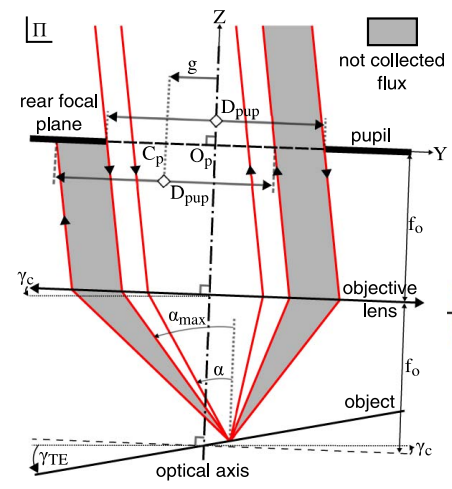

(a)

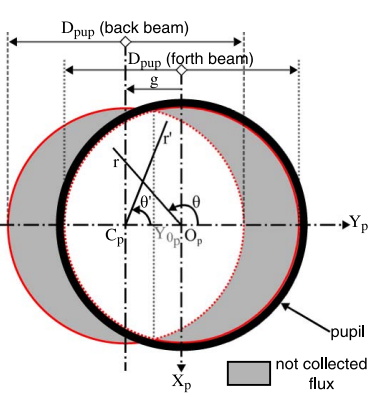

(b)
Fig. 8. (a) Ray tracing illustrating the light collection as a function of the tilt of the sample. (b) Collected light in the plane of the pupil.
For $\gamma_{\mathrm{TE}} \geq 0$

$$
\begin{aligned}
F\left(\gamma_{\mathrm{TE}}, \gamma_{c}, \mathrm{NA}, m\right)= & \left\{2 \int_{\theta_{1}}^{\pi} \int_{\alpha_{1}}^{\alpha_{2}(\theta)} f_{1}\left(\gamma_{\mathrm{TE}}, \alpha, m\right) \mathrm{d} \theta \mathrm{d} \alpha\right. \\
& \left.+2 \int_{0}^{\theta_{2}} \int_{\alpha_{1}}^{\alpha_{3}\left(\theta^{\prime}\right)} f_{1}\left(\gamma_{\mathrm{TE}}, \alpha, m\right) \mathrm{d} \theta^{\prime} \mathrm{d} \alpha\right\} \\
& /\left\{2 \int_{\theta_{1}}^{\pi} \int_{\alpha_{1}}^{\alpha_{2}(\theta)} f_{2}(\alpha) \mathrm{d} \theta \mathrm{d} \alpha\right. \\
& \left.+2 \int_{0}^{\theta 2} \int_{\alpha_{1}}^{\alpha_{3}\left(\theta^{\prime}\right)} f_{2}(\alpha) \mathrm{d} \theta^{\prime} \mathrm{d} \alpha\right\},
\end{aligned}
$$

and for $\gamma_{\mathrm{TE}} \leq 0$,

$$
\begin{aligned}
F\left(\gamma_{\mathrm{TE}}, \gamma_{c}, \mathrm{NA}, m\right)= & \left\{2 \int_{0}^{\theta_{1}} \int_{\alpha_{2}(\theta)}^{\alpha_{1}} f_{1}\left(\gamma_{\mathrm{TE}}, \alpha, m\right) \mathrm{d} \theta \mathrm{d} \alpha\right. \\
& \left.+2 \int_{\theta_{2}}^{\pi} \int_{\alpha_{3}\left(\theta^{\prime}\right)}^{\alpha_{1}} f_{1}\left(\gamma_{\mathrm{TE}}, \alpha, m\right) \mathrm{d} \theta^{\prime} \mathrm{d} \alpha\right\} \\
& /\left\{2 \int_{0}^{\theta_{1}} \int_{\alpha_{2}(\theta)}^{\alpha_{1}} f_{2}(\alpha) \mathrm{d} \theta \mathrm{d} \alpha\right. \\
& \left.+2 \int_{\theta 2}^{\pi} \int_{\alpha_{3}\left(\theta^{\prime}\right)}^{\alpha_{1}} f_{2}(\alpha) \mathrm{d} \theta^{\prime} \mathrm{d} \alpha\right\}
\end{aligned}
$$

with

$$
f_{1}\left(\gamma_{\mathrm{TE}}, \alpha, m\right)=\cos \left[\phi\left(\gamma_{\mathrm{TE}}, \alpha\right)\right] P_{m}(\alpha) \sin \alpha,
$$

$$
f_{2}(\alpha)=P_{1}(\alpha) \sin \alpha,
$$

$$
\theta_{1}=\arccos \left(\frac{2 Y_{0_{p}}}{D_{\text {pup }}}\right),
$$

$$
\theta_{2}=\arccos \left(\frac{-2 Y_{0_{p}}}{D_{\text {pup }}}\right)
$$

$$
\alpha_{1}=\arcsin \left(\frac{-Y_{0_{p}}}{f_{o}}\right),
$$

$$
\alpha_{2}(\theta)=\arcsin (-\mathrm{NA} \cos \theta)
$$

$$
\alpha_{3}\left(\theta^{\prime}\right)=\arcsin \left(-\mathrm{NA} \cos \theta^{\prime}-\frac{2 Y_{0_{p}}}{f_{o}}\right),
$$

$$
Y_{0_{p}}=\frac{g}{2}=-\frac{D_{\text {pup }}}{2} \frac{1+\mathrm{NA}^{2}}{2 \mathrm{NA}}\left(\gamma_{\mathrm{TE}}+\gamma_{c}\right)+o\left(\gamma_{\mathrm{TE}}^{2}, \gamma_{c}^{2}\right) .
$$

$D_{\text {pup }}$ is the diameter of the pupil. $g$ and $Y_{0_{p}}$ are defined in $\left(O_{p} X_{p} Y_{p}\right)$, a plane in the rear focal plane, orientated as $\left(\mathrm{O}_{2} X Y\right)$ and with $O_{p}$ the center of the pupil. $g$ is the distance between $O_{p}$ and the center of the reflected beam $C_{p}$, which depends on $\gamma_{\mathrm{TE}} . Y_{0_{p}}$ is 
the center of $\left[O_{p} C_{p}\right] \cdot \gamma_{c}$ describes a perturbation on the collected flux [see Fig. 8(a)]. It is used to take into consideration negligible phenomena not present in the model. Among other things, this angle is related to the distance between the pupil and the rear focal plane, the light beam divergence, and the decentering of the beam with respect to the optical axis:

$$
\sin (\alpha)=\frac{r \cos (\theta)}{f_{o}}=\frac{r^{\prime} \cos \left(\theta^{\prime}\right)-g}{f_{o}} .
$$

$\left(O_{p} r \theta\right)$ and $\left(C_{p} r^{\prime} \theta^{\prime}\right)$ are two cylindrical coordinate systems [see Fig. 8(b)] used for the integration. $P_{m}$ is the apodization function; it corresponds to the repartition of light on the pupil. There are many possible choices for this function [25], and one chooses

$$
P_{m}(\alpha)=[\cos (\alpha)]^{m} .
$$

$m$ is a parameter used to describe the pupil illumination. In Eqs. (A5) and (A6), the function $P_{1}$ appears in the denominator, for the weighting of $F$. It is possible to choose any value of $m$, but using $m=1$, the denominator is analytically integrable so that the computation time in the calibration process decreases noticeably.

\section{References}

1. W. C. Oliver and G. M. Pharr, "An improved technique for determining hardness and elastic modulus using load and displacement sensing indentation experiments," J. Mater. Res. 7, 1564-1583 (1992).

2. U. Rabe, S. Amelio, E. Kester, V. Scherer, S. Hirsekorn, and W. Arnold, "Quantitative determination of contact stiffness using atomic force acoustic microscopy," Ultrasonics 38, 430-437 (2000).

3. B. Cretin and F. Sthal, "Scanning microdeformation microscopy," Appl. Phys. Lett. 62, 829-831 (1993).

4. B. Cretin and P. Vairac, "Measurement of cantilever vibrations with a new heterodyne laser probe-application to scanning microdeformation microscopy," Appl. Phys. A. 66, S235-S238 (1998).

5. P. Vairac and B. Cretin, "Electromechanical resonator in scanning microdeformation microscopy: theory and experiment," Surf. Interface Anal. 27, 588-591 (1999).

6. P. Vairac, S. Ballandras, and B. Cretin, "Finite element analysis of the behavior of the scanning microdeformation microscope," IEEE Trans. Ultrason. Ferroelectr. Freq. Control 48, 895-899 (2001).

7. J. Le Rouzic, P. Delobelle, P. Vairac, and B. Cretin, "Comparison of three different scales techniques for the dynamic mechanical characterization of two polymers (PDMS and SU8)," Eur. Phys. J. Appl. Phys. 48, 11201 (2009).

8. M. J. Bamber, K. E. Cooke, A. B. Mann, and B. Derby, "Accurate determination of Young's modulus and Poisson's ratio of thin films by a combination of acoustic microscopy and nanoindentation," Thin Solid Films 398-399, 299-305 (2001).

9. R. Feng and R. J. Farris, "The characterization of thermal and elastic constants for an epoxy photoresist SU8 coating," J. Mater. Sci. 37, 033509 (2002).

10. D. C. Hurley and J. A. Turner, "Measurement of Poisson's ratio with contact-resonance atomic force microscopy," J. Appl. Phys. 102, 033509 (2007).

11. J. Le Rouzic, P. Delobelle, B. Cretin, P. Vairac, and F. Amiot, "Simultaneous measurement of Young's modulus and Poisson's ratio at microscale with two-modes scanning microdeformation microscopy," Mater. Lett. 68, 370-373 (2012).

12. M. Françon and S. Mallick, "Compensated polarization interferometers for the observation of phase objects," in Polarization Interferometers: Applications in Microscopy and Macroscopy (Wiley-Interscience, 1971), pp. 55-67.

13. F. Amiot and J. P. Roger, "Nomarski imaging interferometry to measure the displacement field of micro-electro-mechanical systems," Appl. Opt. 45, 7800-7810 (2006).

14. M. J. Boussinesq, "Valeurs des déplacements, des déformations et de pressions intérieures, quand les potentiels se réduisent à un seul de leurs éléments," in Application des potentiels à l'étude de l'équilibre et du mouvement des solides élastiques (Gauthier-Villars, 1885), pp. 81-108 (in French).

15. A. Boisen, S. Dohn, S. S. Keller, S. Schmid, and M. Tenje, "Cantilever-like micromechanical sensors," Rep. Prog. Phys. 74, 036101 (2011).

16. D. Malacara and O. Harris, "Interferometric measurement of angles," Appl. Opt. 9, 1630-1633 (1970).

17. G. D. Chapman, "Interferometric angular measurement," Appl. Opt. 13, 1646-1651 (1974).

18. P. Shi and E. Stijns, "New optical method for measuring smallangle rotation," Appl. Opt. 27, 4342-4346 (1988).

19. C. J. Tay, C. Quan, S. H. Wang, and H. M. Shang, "Determination of a micromirror angular rotation using laser interferometric method," Opt. Commun. 195, 71-77 (2001).

20. W. Gao, P. S. Huang, T. Yamada, and S. Kiyono, "A compact and sensitive two-dimensional angle probe for flatness measurement of large silicon wafers," Precis. Eng. 26, 396-404 (2002).

21. M. Xiao, S. Jujo, S. Takahashi, and K. Takamasu, "Nanometer profile measurement of large aspheric optical surface by scanning deflectometry with rotatable devices-Uncertainty propagation analysis and experiments," Precis. Eng. 36, 91-96 (2012).

22. Y. Surrel, N. Fournier, M. Grédiac, and P.-A. Paris, "Phasestepped deflectometry applied to shape measurement of bent plates," Exp. Mech. 39, 66-70 (1999).

23. J.-R. Lee, J. Molimard, A. Vautrin, and Y. Surrel, "Digital phase-shifting grating shearography for experimental analysis of fabric composites under tension," Compos. Part A Appl. Sci. Manufact. 35, 849-859 (2004).

24. M. Born, E. Wolf, and A. B. Bhatia, "Geometrical theory of optical imaging," in Principles of Optics: Electromagnetic Theory of Propagation, Interference and Diffraction of Light (Cambridge University, 1959), pp. 133-202.

25. A. Dubois, J. Selb, L. Vabre, and A. C. Boccara, "Phase measurements with wide-aperture interferometers," Appl. Opt. 39, 2326-2331 (2000). 